Article

\title{
Virtual Sensing Directional Hub MAC (VSDH-MAC) Protocol with Power Control
}

\author{
Arnold Chau ${ }^{1}$, John Dawson ${ }^{1}(1)$, Paul Mitchell ${ }^{1, *}$ and Tian Hong Loh ${ }^{2}$ \\ 1 Department of Electronic Engineering, University of York, York YO10 5DD, UK; \\ arnold.chau@york.ac.uk (A.C.); john.dawson@york.ac.uk (J.D.) \\ 2 Electromagnetic \& Electrochemical Technologies Department, National Physical Laboratory, Hampton Road, \\ Teddington, Middlesex TW11 0LW, UK; tian.loh@npl.co.uk \\ * Correspondence: paul.mitchell@york.ac.uk
}

Received: 8 June 2020; Accepted: 27 July 2020; Published: 29 July 2020

check for updates

\begin{abstract}
Medium access control (MAC) protocols play a vital role in making effective use of a multiple access channel as it governs the achievable performance such as channel utilization and corresponding quality of service of wireless sensor networks (WSNs). In this paper, a virtual carrier sensing directional hub (VSDH) MAC protocol incorporating realistic directional antenna patterns is proposed for directional single hub centralized WSNs. While in most instances, MAC protocols assume idealized directional antenna patterns, the proposed VSDH-MAC protocol incorporates realistic directional antenna patterns to deliver enhanced link performance. We demonstrate that the use of directional antennas with a suitable MAC protocol can provide enhanced communication range and increased throughput with reduced energy consumption at each node, compared to the case when only omnidirectional antennas are used. For the scenarios considered in this study, results show that the average transmit power of the sensor nodes can be reduced by a factor of two, and at the same time offer significantly extended lifetime.
\end{abstract}

Keywords: medium access control (MAC); wireless sensor network (WSN); wireless communication; directional antennas; energy efficiency; power control

\section{Introduction}

Wireless sensor networks (WSNs) have been employed in a wide range of health care, industrial and environmental monitoring applications [1-3]. For many applications, the use of batteries in sensor nodes places constraints on the energy budget, so it is important to maximize the performance of the network whilst minimizing the sensor node energy consumption. Interference, lack of fairness and energy consumption are the key constraints in WSNs, which poses challenges to the design of medium access control (MAC) protocols. Directional antennas provide the potential to increase transmission range and/or reduce transmission power, to reduce interference along with the prospect of allowing spatial reuse. In order to make the best use of directional antennas, suitable MAC protocols must be designed.

On selection of suitable MAC protocols for WSNs, one could consider either contention-based or contention-free protocols. Contention-based protocols can be less efficient than those without contention in terms of throughput performance for large star topologies due to the large number of collisions when the data traffic offered load is high. However, they are simpler and typically provide lower delay in smaller WSNs [4]. Contention-based protocols are a promising approach for directional MAC protocols, as they enable multiple nodes to simultaneously access a channel without the need for synchronization. Scheduling and synchronization are the main challenges for contention-free protocols, especially for WSNs with mobile nodes and/or a varying number of nodes. 
In [5], we proposed a simple directional hub MAC protocol for star topology WSNs based on the Pure Aloha protocol, in which the performance differences between a realistic directional antenna pattern and idealized directional antenna pattern were demonstrated. It is shown that although directional antennas can provide high throughput performance, the antenna pattern may still have a significant effect on spatial reuse and network performance. In this proposed protocol, node complexity and power consumption are minimized by having only a single omnidirectional antenna on the basic sensor nodes. The hub carries multiple directional antennas and can be continually powered as its complexity and power consumption are not considered critical compared with the basic sensor nodes. Energy consumption and fairness were considered in [6], in which a MAC protocol with transmit power control on nodes was analyzed. The performance enhancement that can be achieved by the use of power control and directional hub antennas was demonstrated, in terms of network throughput, node power consumption, and fairness.

Most previous works on the use of directional antennas have assumed the use of idealized antenna patterns where each antenna beam is distinct, with no overlap with adjacent beams and having a constant antenna gain across the beam [7-15]. Some work has assumed that the nodes are capable of knowing each other's position [7,9-11] or that nodes have complex, steerable antennas [7,12]. In addition, multiple antennas are often required at the nodes as well as the hub $[7-13,16]$ which increases both the complexity and energy consumption of the nodes. Some of the protocols proposed also require multiple channels to successfully operate $[8,13,16]$. Only a few papers within the literature $[14,17-19]$ have considered the energy consumption of the protocol, which is an important factor for low power nodes [20].

In this paper, a modified directional CSMA/CA (carrier sense multiple access with collision avoidance) protocol is proposed, which is similar to the IEEE 802.11 WI-FI standard and the IEEE 802.15.4 standard for WSNs. A version without the traditional physical carrier sensing is used (to reduce energy consumption). Here virtual carrier sensing is performed via handshaking packets. A version with physical carrier sensing similar to the CSMA/CA protocol is also considered for comparison. The hub node is equipped with multiple directional antennas, and the channel is efficiently utilized through the benefits of spatial reuse. A dynamic transmit power control algorithm is employed at the wireless sensor nodes to improve node energy efficiency. A uniform signal-to-interference-plus-noise ratio (SINR) is achieved for packets from all sensor nodes in the network. As shown through simulations, the proposed protocol leads to improvements in network throughput, energy consumption, and fairness performance. The effects of antenna pattern overlap are also significantly reduced by the proposed protocol.

The rest of the paper is organized as follows: In Section 2, we describe the network topology and proposed MAC protocol in detail. In Section 3, we describe the simulation methodology and in Section 4, we present results that show the improved throughput and performance that can be achieved, along with the effect of the antenna gain, power control algorithm, and antenna pattern.

\section{Medium Access Control Protocol}

This section outlines the operation of the virtual sensing directional hub MAC protocol (VSDH-MAC) and the carrier sensing version (DIFS-VSDH-MAC) named after the DCF Interframe Space (DIFS), the period during which carrier sensing occurs, in the IEEE 802.11 CSMA/CA/DCF.

The proposed VSDH-MAC protocol is similar to the IEEE 802.11 DCF (distributed coordination function), which uses CSMA/CA/DCF protocol [21], and the IEEE 802.15.4 protocol which is a CSMA/CA protocol. However, continuous physical channel sensing is not performed. Instead, virtual channel sensing is enabled using request-to-send/clear-to-send (RTS/CTS) packets in a similar way to the CSMA/CA/DCF protocol. The packet exchange procedure of the VSDH-MAC protocol follows the IEEE 802.11 CSMA/CA/DCF method with the RTS/CTS and DATA/ACK (data/acknowledgement) packet structure. 
In this paper, we consider a network with a single hub node which has a number of directional antennas which can operate simultaneously, each in half-duplex (HDX) mode. The power consumption of the hub node is not considered to be constrained. The sensor nodes are assumed to have a single omni-directional antenna to reduce hardware complexity. It is assumed that the transmit power of each sensor node can be adjusted to minimize the transmit power consumption and inter-node interference. Furthermore, we assume that all communications are initiated by the sensor nodes so that they can remain quiescent and minimize energy consumption when they have no data to transmit.

\section{VSDH-MAC Channel Access Algorithm}

When a node has no data packet to transmit, i.e., its packet queue is empty, it will remain in sleep state to conserve energy. When a node wishes to transmit a data packet, it will send a short RTS packet to the hub immediately, using its maximum transmit power. The maximum transmit power is used because we have assumed sensor nodes might move, and we require the RTS to reach the hub regardless of the current node position, which is assumed unknown. Extensive simulations implemented in Riverbed Modeler have shown that although the RTS packets are sent with maximum power, there is no significant impact to the node energy consumption and overall network throughput. If it receives a CTS packet from the hub, in response to the RTS packet, it may then transmit a data packet to the hub. The node is assumed to know the hub transmit power and uses the received power of the CTS to compute the path loss and thereby choose the least required packet transmit power to successfully transmit the data packet, assuming a reciprocal channel. This is done in order to minimize both the interference to other nodes and the node power consumption, although it is of course simple to introduce an appropriate link margin by increasing the transmit power above the calculated minimum if desired, to account for uncertainties and variation in the channel, e.g., due to shadowing. In this study, we assumed the same background noise at both the transmitter and receiver. In a real network, while the reciprocal path is the same, the noise might not. Hence, in a practical protocol it would be necessary for the hub to calculate the required transmit power with its background noise and include the value in the CTS as a reference for the sensor node. RTS and CTS packets both contain a network allocation vector (NAV) which defines the time required to complete the subsequent data packet transmission and associated handshaking. Other nodes hearing a CTS above a certain amplitude threshold will delay their transmission to avoid collision. The threshold is defined as the product of the packet transmit power and 2.1 the receiving antenna gain at angle $\theta\left(G_{\theta}\right)$, where $\theta=\frac{360}{M}$, and $M$ is denoted as the number of directional antenna at the hub. Nodes only listen for a CTS during the time when they are awaiting a reply for their own RTS. This also maximizes the chance of avoiding collisions between active nodes, whilst minimizing node energy consumption, as a node does not need to listen for a CTS except when it is likely to be transmitting data. After sending an RTS the node waits for a time slightly larger than the expected round trip time (RTT). If a node receives no response to its RTS within this time, it will enter a back-off state, which delays transmission of another RTS for the same data by a random delay in the range [0, $C W-1]$ where $C W$ is an interval called the Contention Window. Subsequent failures to receive a CTS increase the back-off time range exponentially by a factor of two in each case. The value of the random back-off interval is chosen from the $C W$, which lies between two preconfigured values, $C W \_$min and $C W \_\max$. The values for these are identical to the IEEE 802.11 CSMA/CA/DCF protocol. The contention window is set to CW_min at the first transmission attempt, and doubles after each unsuccessful attempt, until it reaches $C W \_m a x$. The contention window is reset to $C W \_m i n$ after every successful transmission. After the counter reaches $C W \_$max the packet transmission would be abandoned, and the error would also be reported to the layer above. Once a packet is transmitted, if an acknowledgment is not received within the specified RTT time for the data packet, a re-transmission with maximum transmission power for the data packet will be performed following the same RTS/CTS/DATA/ACK sequence. Thus, the node protocol is designed to require minimal electrical and processing power. 
Operation of the protocol at the hub is slightly more complex as it has multiple antennas and corresponding transceivers. The hub algorithm differs in the following manner. It is assumed to be capable of communicating via all antennas simultaneously and listening continuously from any that is not transmitting. It is assumed that the hub will not initiate a transmission to the node. If transmission of messages from the hub to the nodes is required, it can be included in the ACK packet at the end of each exchange. If the hub receives an RTS on one or more antennas from a node it will note which of the antennas provides the highest SINR and use that antenna for subsequent communications with the node until a packet arrives at a different antenna with higher SINR from the same node. If the hub has received the same packet from multiple antennas with equal SINR, then the subsequent transmission will use a random antenna selection between them until an optimum antenna is established. When an RTS is received from a node, and if no other RTS has reserved the optimum antenna, the hub will reserve the optimum antenna for a period indicated as NAV (network allocation vector) in the RTS (NAV) and then transmit a CTS to the node from the optimum antenna. The CTS also contains a NAV which will cause any listening node to delay its transmission. As nodes do not continuously listen there is still a probability of collision by a node that does not hear the ongoing exchange when it is ready to transmit. Algorithm 1 summaries the steps of the proposed virtual sensing approach for WSNs with a directional hub node.

A modified VSDH-MAC protocol with an additional physical channel sensing (DIFS long) is also considered in this paper (DIFS-VSDH-MAC), in which nodes sense the channel prior to transmission of an RTS. If any signal above the SIR threshold is received, it will pause the DIFS counter and enter the back-off stage according to the NAV. This improves the overall throughput performance of the protocol $y$ reducing the probability of RTS/CTS collision, at the cost of increased sensor node energy consumption and transmission delays.

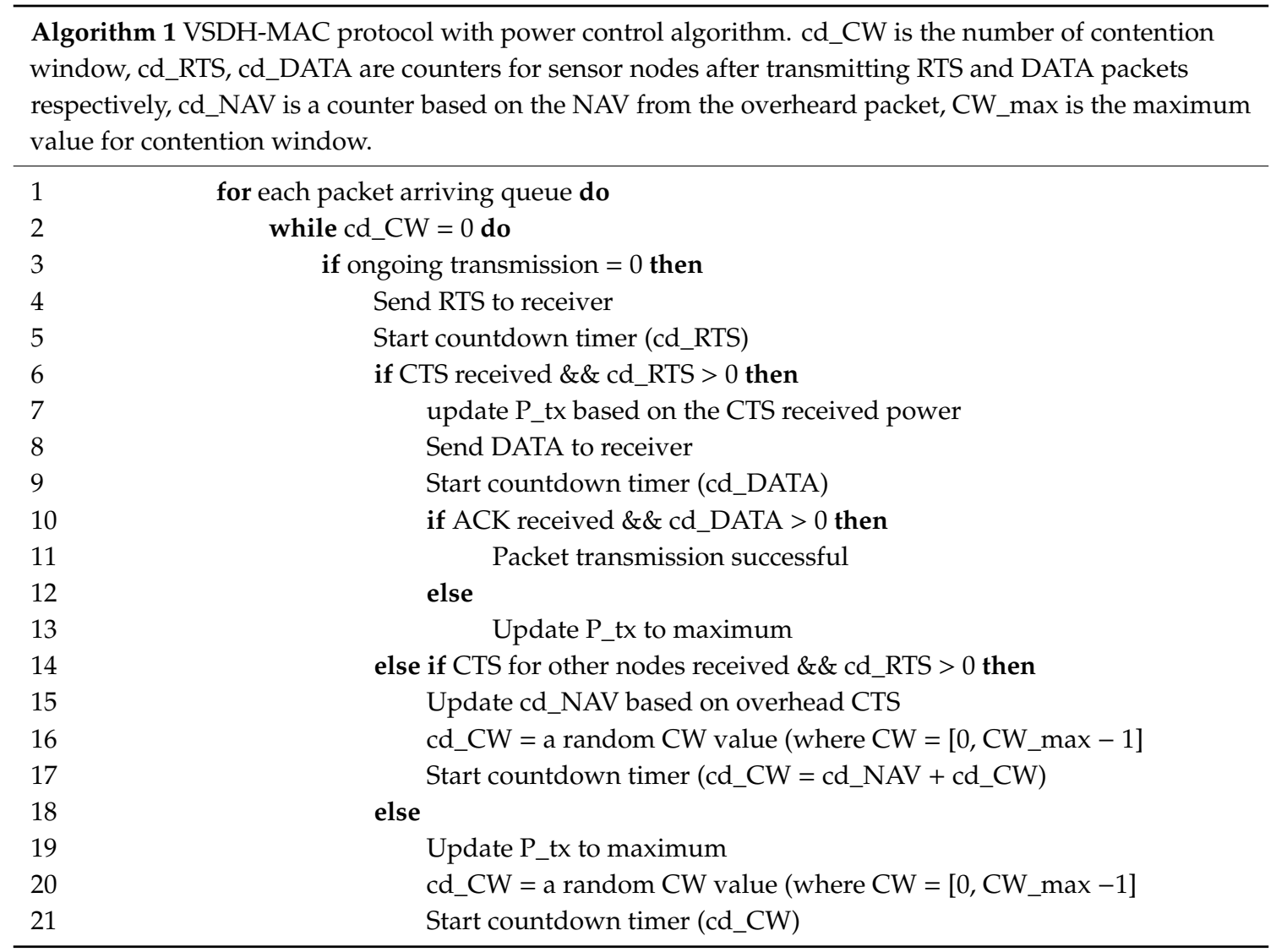




\section{Simulation Methodology}

\subsection{Network Configuration}

To fairly characterize the performance of the protocols, a series of randomly generated configurations are considered, and an example is shown in Figure 1. We chose a single hop star topology with half duplex (HDX) operation on a single frequency channel as this is simple and common in WSNs. A HDX operation is defined as a system supporting communication in both directions, but only one direction at a time. A two-dimensional distribution of sensor nodes is considered in the study. The star topology allows for a continuously powered hub where energy usage and complexity are not considered to be an issue. By adding directional antennas to the hub, we can improve throughput, and range or energy consumption. We consider $n$ nodes randomly distributed in a $100 \times 100 \mathrm{~m}^{2}$ grid, where the $\mathrm{x}$ and $\mathrm{y}$-coordinates are each chosen using a pseudorandom number generator with a uniform distribution between plus and minus $50 \mathrm{~m}$. The single hub base station node is positioned at the center of the grid.

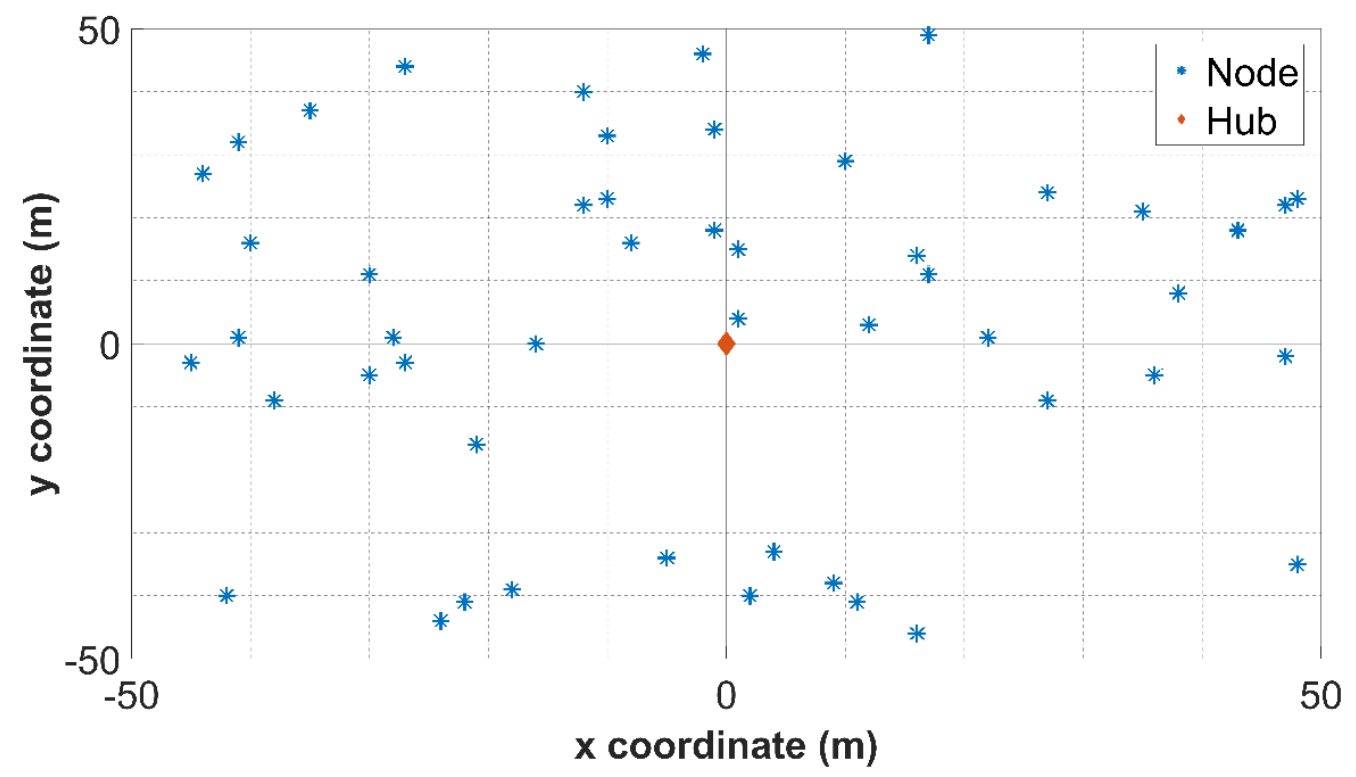

Figure 1. An example of centralized wireless sensor networks (WSNs) topology.

\subsection{Simulation Setup}

To evaluate the performance of the proposed protocol, simulations have been performed using Riverbed Modeler (formerly known as OPNET) [22]. In all simulations, we consider only free space propagation as an illustrative example. We chose to use four antennas as a reasonably practical number to illustrate the performance of a multi-antenna hub. Fewer antennas could be used with litter effort. However, if a significant increase in the number of antennas were required, the issue of beam overlap may become a significant problem. Some overlap is necessary as it is not possible to design antennas with ideal cutoff at the beam edges, but as described in [5], beam overlap is a significant factor in limiting the throughput performance. As the sector angle decreases with increasing numbers of antennas, the degree of overlap must be reduced by the same amount to maintain the same performance per antenna. We suspect this will create some practical difficulties in antenna design and alignment.

The transmission parameters are shown in Table 1. Note that SIFS and BPSK, in Table 1 stand for short interframe space and binary phase shift keying, respectively. The simulator uses the SINR to determine the bit error rate (BER). This BER value is used to determine if each individual bit is received in error, assuming randomly distributed errors. A uniformly distributed random number between zero and one is generated randomly. This number is compared with the BER threshold (obtained from 
a look up table of SINR vs BER for a given modulation scheme), and one or more bit errors will result in a discarded packet. Packets with errors are rejected by the protocol. The data packets are generated according to a Poisson process with a rate $(G)$, which is referred as the channel offered load or traffic load. The Poisson arrival process gives an exponentially distributed inter-arrival time of the data packet generation.

Table 1. Transmission parameters.

\begin{tabular}{cc}
\hline Parameters & Values \\
\hline Frequency band & $2.4 \mathrm{GHz}$ \\
Channel bit rate & $250 \mathrm{kbit} / \mathrm{s}$ \\
RTS, CTS, ACK length & $8 \mathrm{bits}$ \\
Data length & $1024 \mathrm{bits}$ \\
Number of Hub Antenna $(M)$ & 4 \\
Maximum Transmission Power & $0.052 \mathrm{~W}$ \\
Node Received Power & $0.059 \mathrm{~W}$ \\
Node Sleep Power & $0003 \mathrm{~mW}$ \\
Digital modulation & BPSK \\
CW_min & 31 \\
CW_max & 1023 \\
SIFS & $10 \mathrm{us}$ \\
\hline
\end{tabular}

\subsection{Directional Antennas}

In order to demonstrate the effect of antenna pattern on performance, simulations were performed with two real antenna patterns. Antenna 1 (Ant 1) is based on a 3 element Yagi design and the second antenna (Ant 2) is based on the low cost antenna from [23], as demonstrated in Figure 2. In addition, we consider an ideal sectored antenna with uniform gain over a $90^{\circ}$ sector and zero elsewhere as commonly used in other studies. Detailed discussion on SIR limit analysis can be found in [5].

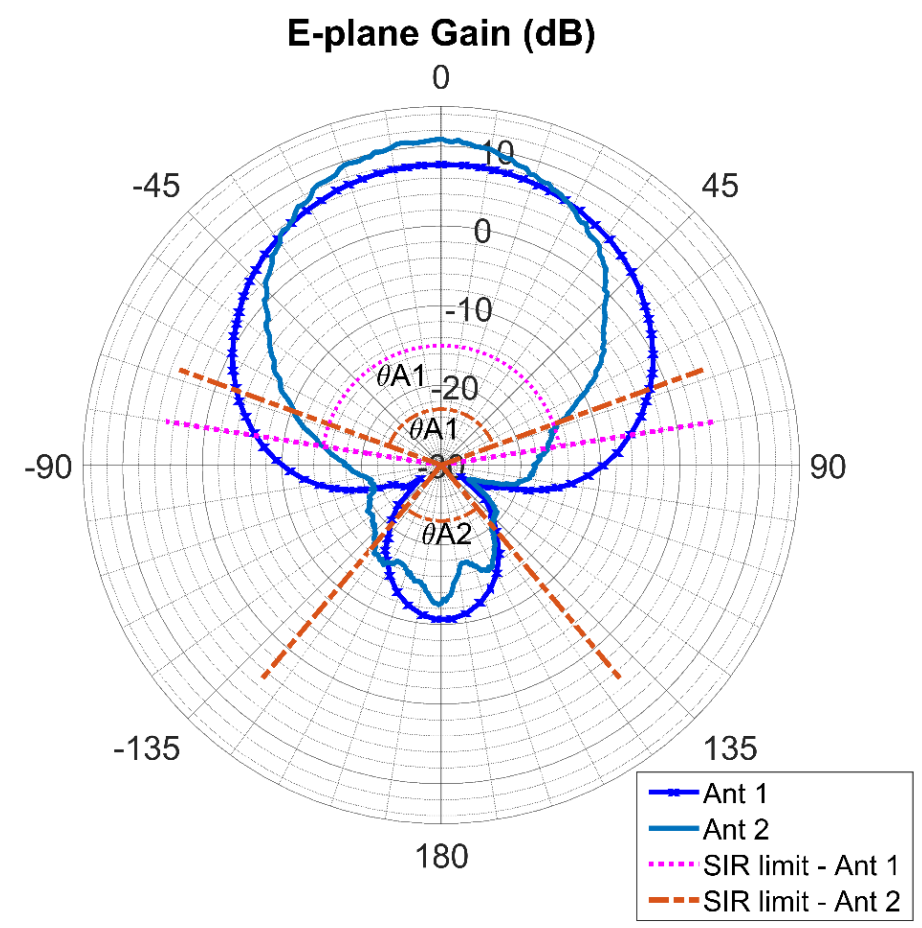

Figure 2. Polar plot of antenna gain pattern for Ant 1 and Ant 2 with its SIR (signal-to-interference ratio) limit angles, where $\theta_{A}=\theta_{A 1}+\theta_{A 2}$. 


\subsection{Energy Consumption Calculation}

This section provides the average energy consumption analysis of the proposed VSDH-MAC protocol. The average energy consumption during data transmission, reception and control packets is given below: successful data packet transmission $\left(E_{t x}\right)$ :

$$
E_{t x}=P_{t x_{R T S}} T_{R T S}+P_{t x_{D A T A}} T_{D A T A}+P_{r x}\left(T_{C T S}+T_{A C K}+2 x\left(T_{p}+T_{S I F S}\right)\right),
$$

colliding RTS or CTS transmission $\left(E_{C_{-} R T S}\right)$ :

$$
E_{C_{-} R T S}=P_{t x_{R T S}} T_{R T S}+P_{r x}\left(T_{C T S}+T_{p}\right),
$$

colliding DATA or ACK transmission $\left(E_{C_{-} D A T A}\right)$ :

$$
E_{\mathcal{C}_{-} D A T A}=E_{\mathcal{C}_{-} R T S}+P_{t x_{D A T A}} T_{D A T A}+P_{r x}\left(T_{A C K}+T_{p}+T_{S I F S}\right),
$$

back-off due to unsuccessful RTS/CTS communication $\left(E_{B O}\right)$ :

$$
E_{B O}=E_{C_{-} R T S}+P_{\text {sleep }}\left(T_{C W}\right),
$$

overhearing reception destined to other user after RTS transmission, $\left(E_{\mathrm{OH}}\right)$ :

$$
E_{O H}=E_{C_{-} R T S}+P_{r x}\left(T_{\text {SIFS }}\right)+P_{\text {sleep }}\left(T_{N A V}+T_{C W}\right),
$$

sleep when no packet transmission is required $\left(E_{\text {sleep }}\right)$ :

$$
E_{\text {Sleep }}=P_{\text {Sleep }} T_{\text {Sleep }}
$$

in addition, when carrier (DIFS) sensing is used, additional energy $\left(E_{D I F S}\right)$ is consumed:

$$
E_{D I F S}=P_{r x} T_{D I F S},
$$

where $T_{D I F S}$ is the time during which the carrier is sensed. If a transmission is detected during $T_{D I F S}$ then additional energy is expended ( $E_{O H}$ DIFS $)$ whilst the node waits before attempting to transmit again:

$$
E_{O H_{-} D I F S}=E_{D I F S}+P_{\text {sleep }}\left(T_{N A V}+T_{C W}\right),
$$

where, $P_{\text {sleep }}, P_{t x_{R T S}}, P_{t x_{D A T A}}$, and $P_{r x}$ are the power consumed in sleep, transmit and receive mode respectively. $T_{S I F S}, T_{D I F S}$ and $T_{P}$ are the SIFS and DIFS time duration from IEEE 802.11 DCF standard and the propagation time of the packet. $T_{C W}$ is the back-off time duration. $T_{R T S}, T_{C T S}, T_{D A T A}$, and $T_{A C K}$ denotes the packet transmission time for RTS, CTS, DATA, and ACK packets respectively. $T_{\text {sleep }}$ is the time for the node to stay in the sleep state. $T_{N A V}$ represents the back-off time indicated from the received NAV.

Figure 3 shows a breakdown of the average energy consumption per successful data bit in a sensor node with respect to the channel offered load. Figure 3a is the energy consumption of the VSDH-MAC protocol with power control strategy. Figure $3 b$ is the energy consumption of the VSDH-MAC protocol without the power control strategy. Figure $3 \mathrm{c}$ is the energy consumption of the IEEE 802.11 DCF protocol. By comparing those figures, it can be seen that the VSDH-MAC protocol provides a far higher energy efficiency than CSMA/CA protocol. Figure 4 shows the additional transmission required for the DIFS sensing. Table 2 shows the operation states of the sensor node and the power consumption of each state. The values are based on typical figures for current radio modules and serve only for comparative purposes. 


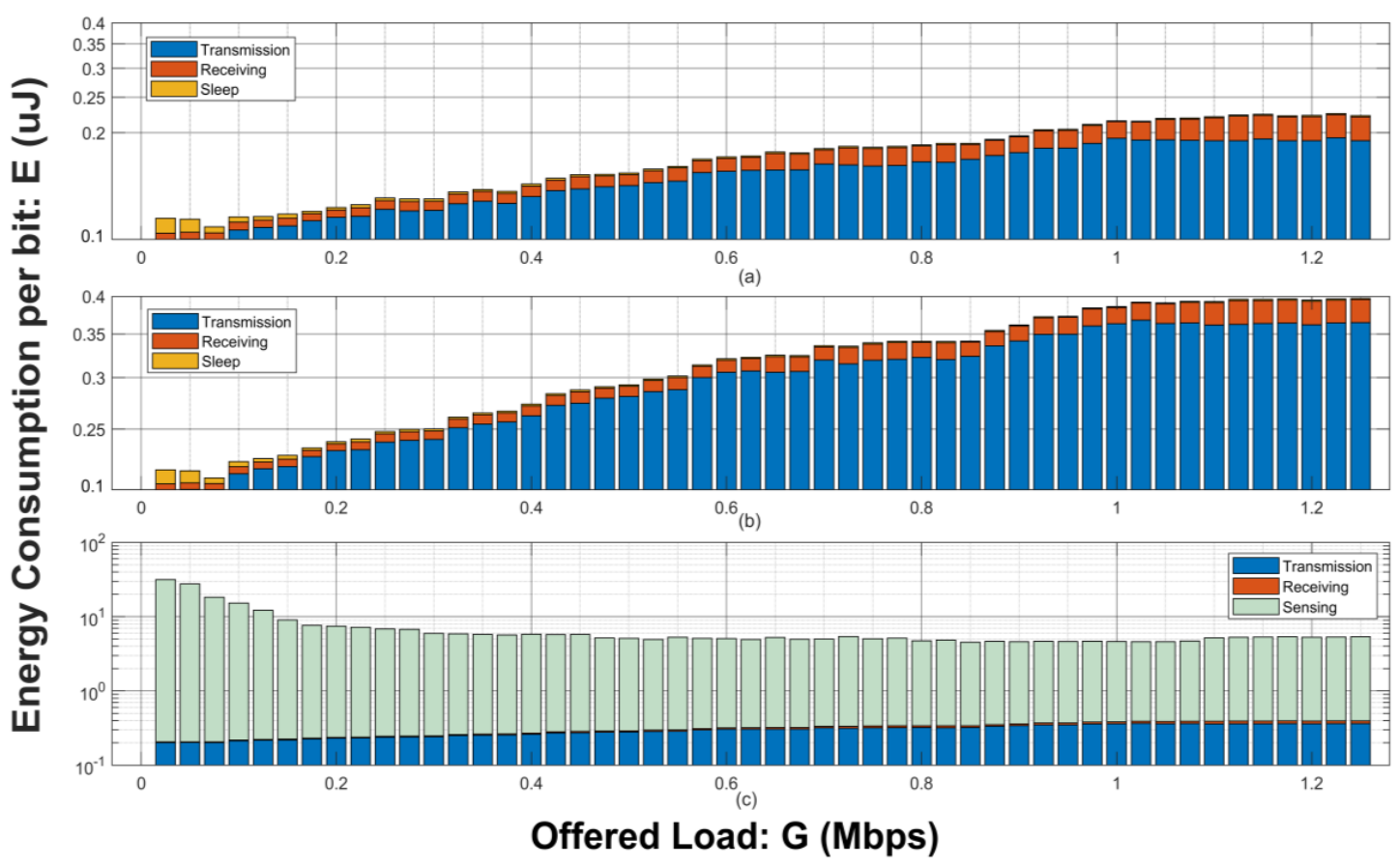

Figure 3. The comparison of required transmission energy per bit virtual sensing directional hub, medium access control protocol (VSDH-MAC) protocol with power control (a) and without power control (b), and carrier sense multiple access with collision avoidance (CSMA/CA)/distributed coordination function (DCF) protocol (c).

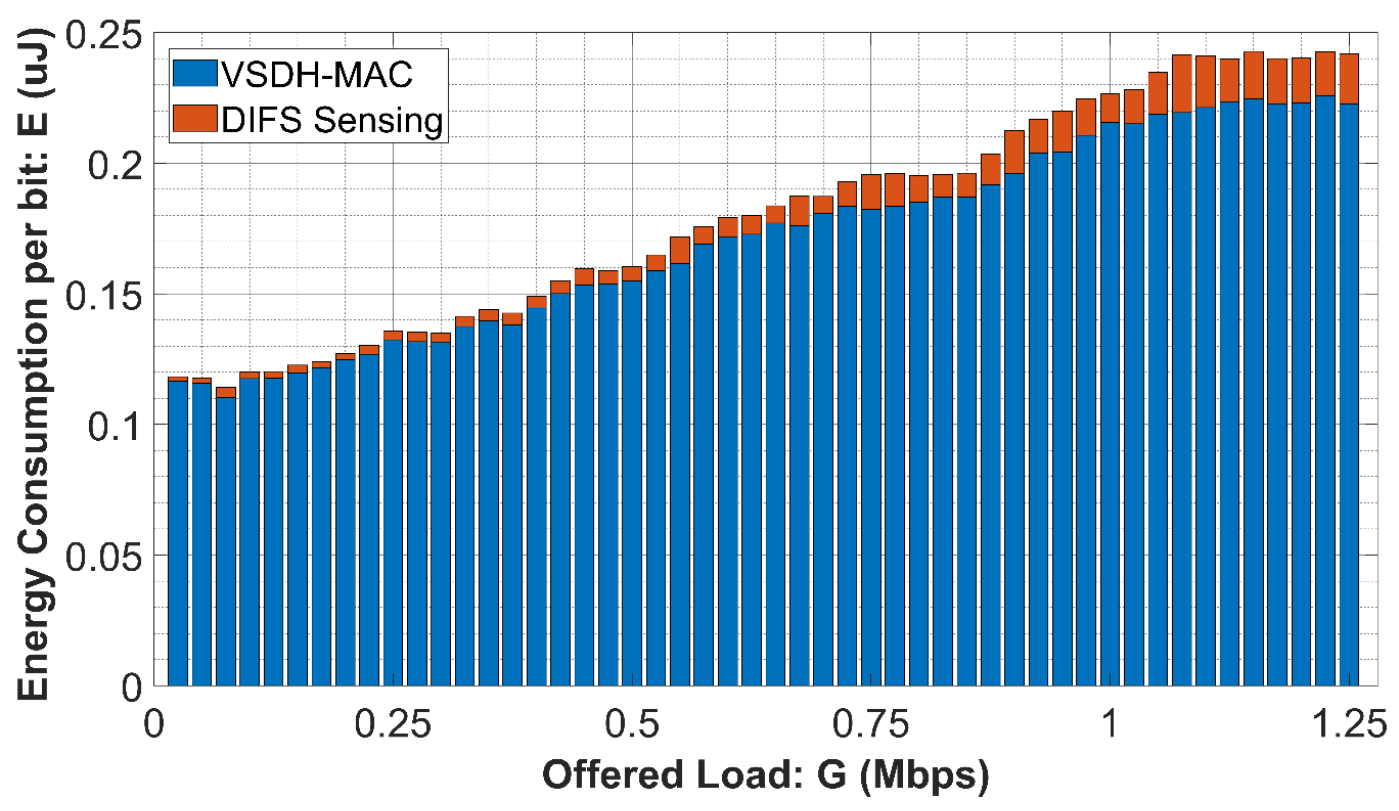

Figure 4. The transmission energy per bit for a four antennas DIFS-VSDH-MAC protocol showing the proportion of energy used by DIFS (DCF Interframe Space) carrier sensing.

Table 2. Operational states for Finite State Machine (FSM) of sensor nodes.

\begin{tabular}{ccccc}
\hline State & Activity & Tx & Rx & Power Required \\
\hline$S_{0}$ & Sleep & Off & Off & $0.003 \mathrm{~mW}$ \\
$S_{1}$ & RTS Tx & On & Off & $52 \mathrm{~mW}$ \\
$S_{2}$ & Receiving & Off & On & $59 \mathrm{~mW}$ \\
$S_{3}$ & Data Tx & On & Off & $26 \mathrm{~mW}$ (Average) \\
\hline
\end{tabular}


We expect the number of hub antennas to have a small impact on the node energy usage in a given scenario. Firstly, as the number of nodes in each sector is reduced there is likely to be fewer collisions which would reduce the energy wasted by this mechanism. In addition, an increased number of antennas would require a narrower beam width per antenna, which implies increased gain in most cases; this would reduce the required transmission power for both the nodes and the hub in a given scenario.

\section{Results Analysis}

The offered load is distributed evenly across all of the sensor nodes. The network throughput is the channel capacity successfully used by all sensor nodes with the maximum of $M$ Erlangs, in which $M$ is the number of directional antennas equipped at the hub. For the purpose of understanding the link performance of the protocol, the results will be expressed as the total number of data bits successfully received per unit time.

Figure 5 shows the throughput of directional hub Aloha (DH-Aloha) protocol [5] averaged over 10 randomly generated networks for each of the three directional antenna types. As predicted in our previous work [5], the antenna pattern has a significant effect on throughput. The idealized antenna pattern with no overlap between sectors, shows a substantially larger throughput than can be achieved with the real antennas with patterns that have some overlap. As depicted in Figure 4, due to the shape of the antenna pattern, the reason that Ant 1 has a higher throughput than Ant 2 is due to the fact than although Ant 2 has a narrower beam-width, it has a larger back lobe. Using the analysis in [5], the back lobe increases the overlapping ratio $(r)$, as $\theta_{A}$ increases. This results in more packet collisions caused by interference from antenna patterns overlapping.

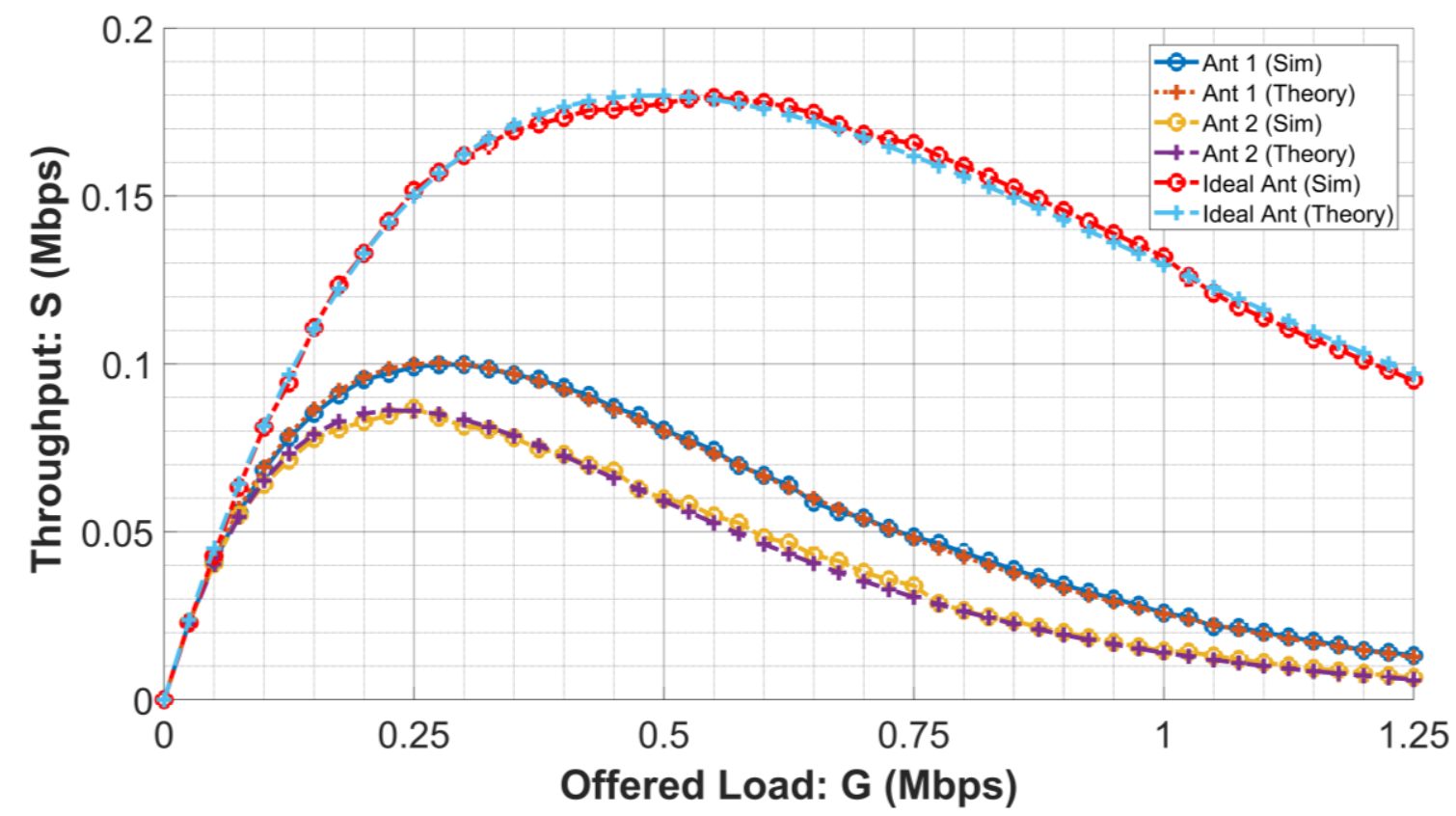

Figure 5. Throughput comparison of different antenna patterns with the DH-Aloha protocol with $M=4$.

In Figure 6, the throughput of the VSDH-MAC protocol for Ant 1 and Ant 2 is presented. The difference between the throughputs of the two antennas are significantly smaller than in Figure 5 . This is because the power control mechanism reduces the effect of antenna pattern overlap by adjusting the node transmission power. The adjusted transmission power reduces the interference caused by the back lobe. 


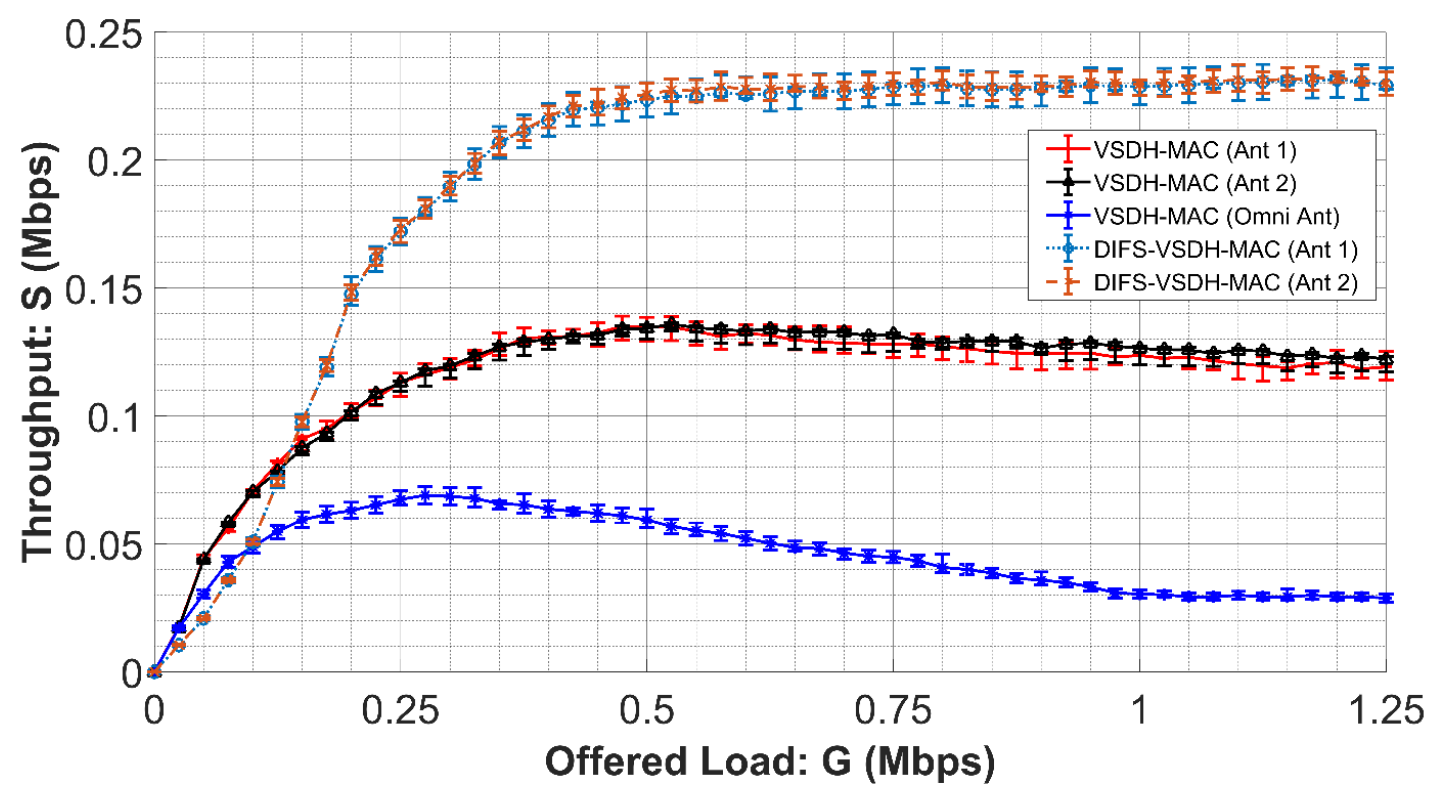

Figure 6. Throughput of the VSDH-MAC and DIFS-VSDH-MAC protocols with different antennas patterns with $M=4$, compared against the VSDH-MAC with a single omni-directional hub antenna.

It is useful to compare the throughput performance of the VSDH-MAC and DIFS-VSDH-MAC protocols with other directional MAC protocols. Two other directional MAC protocols have been replicated for the purpose of performance comparison. Both protocols have been replicated with the parameters described in Section 3. In the DMAC (directional virtual carrier sensing MAC) protocol proposed in [24], the simulation results showed a saturation throughput of $0.225 \mathrm{Mbps}$ with the same simulation setup as in Section 3. However, it is assumed that all nodes are equipped with beamforming directional antennas and global positioning system (GPS). The DMAC protocol is based on the IEEE 802.11 standard and nodes are required to perform channel sensing prior to transmission. The use of GPS and channel sensing has significantly increased the node energy consumption. With idealized antenna patterns, the VSDH-MAC and DIFS-VSDH-MAC protocols can achieve a saturated throughput of $0.336 \mathrm{Mbps}$ and $0.426 \mathrm{Mbps}$, which is approximately a factor of 1.5 or 1.9 higher than the saturated throughput of DMAC. The CMDMAC (cooperative multichannel directional MAC) is a similar protocol that requires multiple channels [25]. It requires one radio channel for control packets such as RTS, CTS and $\mathrm{ACK}$, and a second radio channel for data transmission. Our simulation results indicate a saturated throughput of $0.45 \mathrm{Mbps}$ from the CMDMAC protocol with the same simulation setup as in Section 3 and ideal directional antennas. All sensor nodes must be equipped with an omni-directional antenna for overhead communication and an idealized steerable directional antenna for data transmission. The additional channel provides continuous channel sensing which provides advantages in terms of throughout performance. Although the CMDMAC protocol provides better throughput performance compared with the VSDH-MAC protocol, under these conditions, the additional requirements mean that the throughput performance comes at the cost of increased node manufacturing cost and energy consumption. Moreover, Figure 7 shows that the throughput performance of applying an idealized antenna pattern is significantly higher than using realistic antenna patterns. 


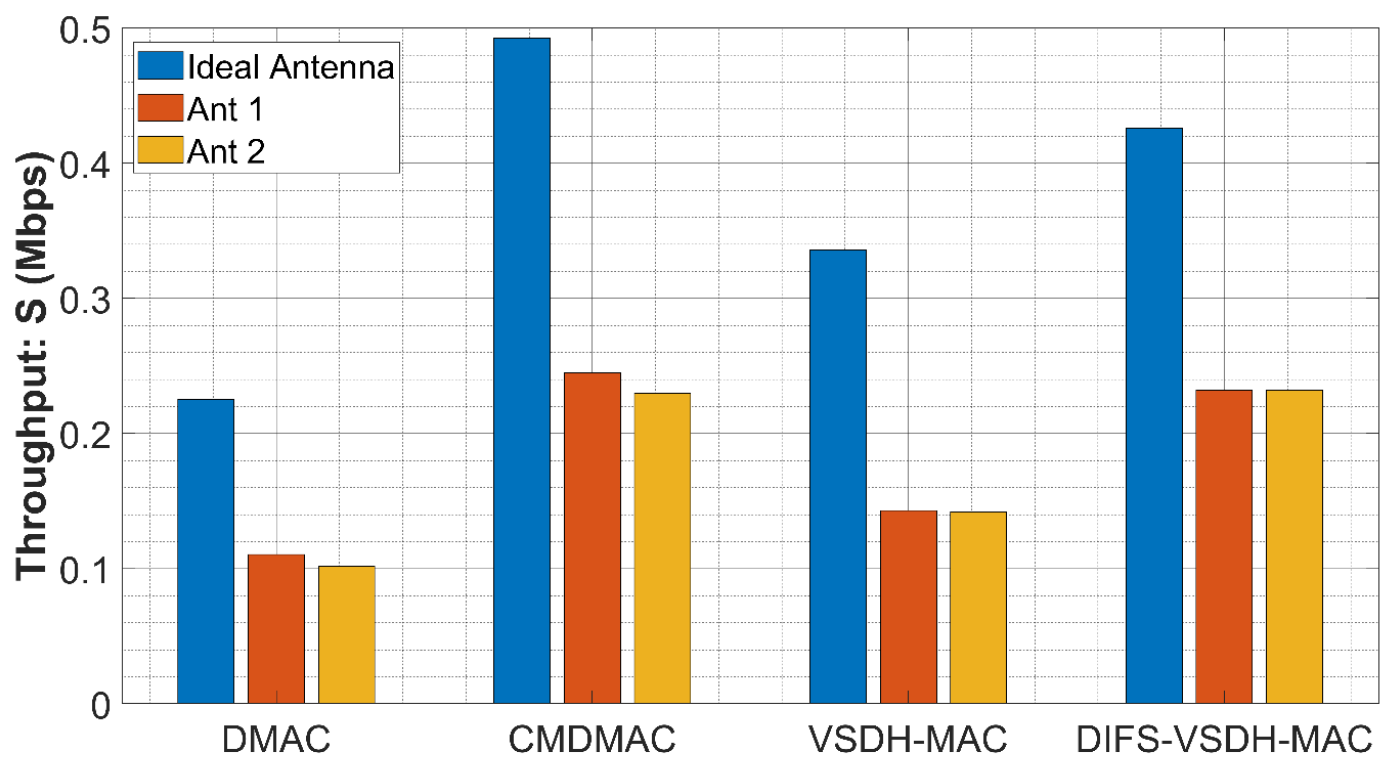

Figure 7. Impact of antenna pattern on throughput performance with $M=4$.

Figure 8 shows the average transmission energy required by the VSDH-MAC protocol with and without power control, for each successful data bit. The power control algorithm can reduce the average required transmission energy by a factor of two. One of the goals of the VSDH-MAC is to prolong the lifetime of the sensor and hence the network lifetime. To quantitatively compare these directional MAC protocols, we adopt the quoted values of current consumption values from MICAz mote [26]. Two 1.5V batteries rated at $2000 \mathrm{mAh}$ each are assumed for each sensor node. We assume the current draw and the size of the packets are fixed. Figure 9 shows the numerical comparison of the expected lifetime obtained from the directional MAC protocols including the directional CSMA/CA. Figures 8 and 9 highlights that the energy efficiency and lifetime expectancy of the VSDH-MAC outperforms the other protocols. Comparing to the VSDH-MAC protocol, the physical carrier sensing from the other directional MAC protocols contribute a significant amount of energy consumption to the sensor nodes. This mechanism with the lack of transmit power control further reduce the lifetime of the sensor nodes. It is important to have an accurate energy model and lifetime estimation of a sensor node, as it directly impacts the lifetime of a WSN.

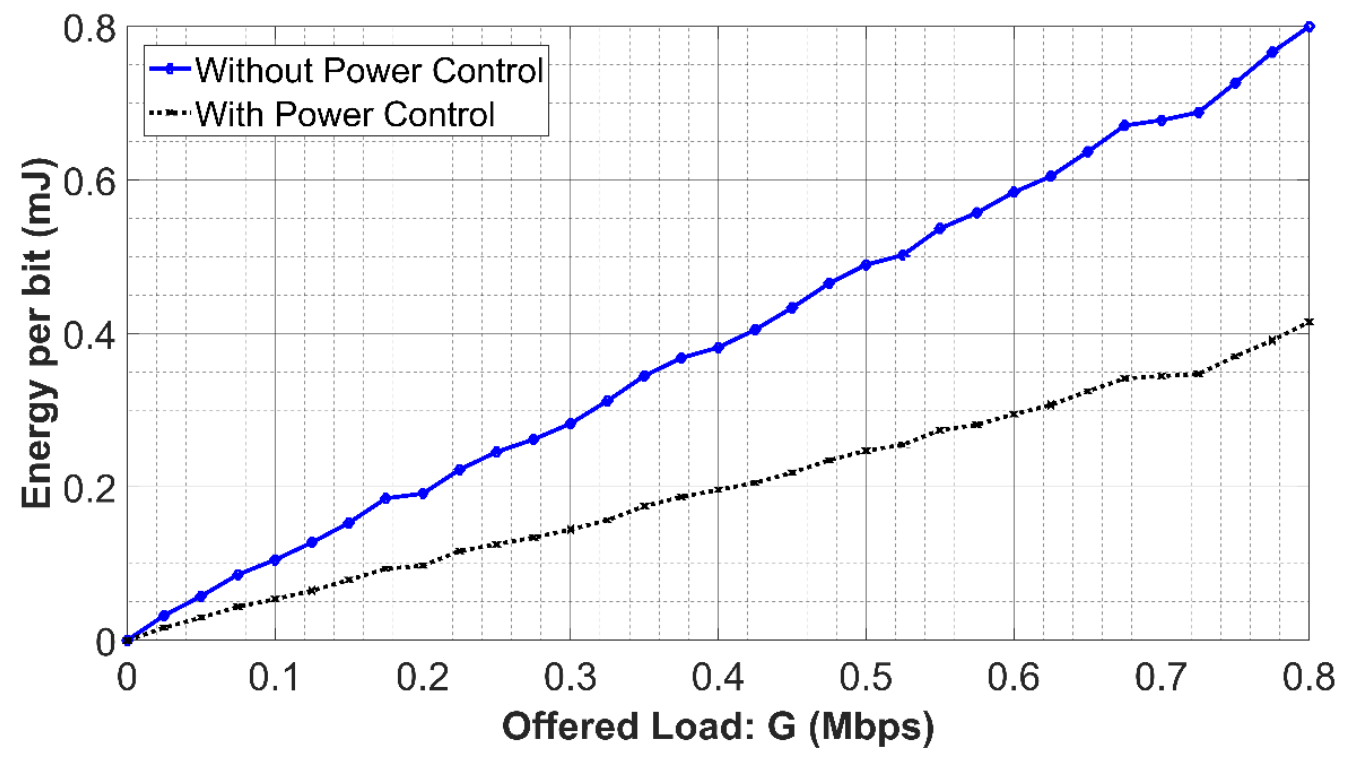

Figure 8. The required transmission energy per bit with and without the proposed power control scheme. 


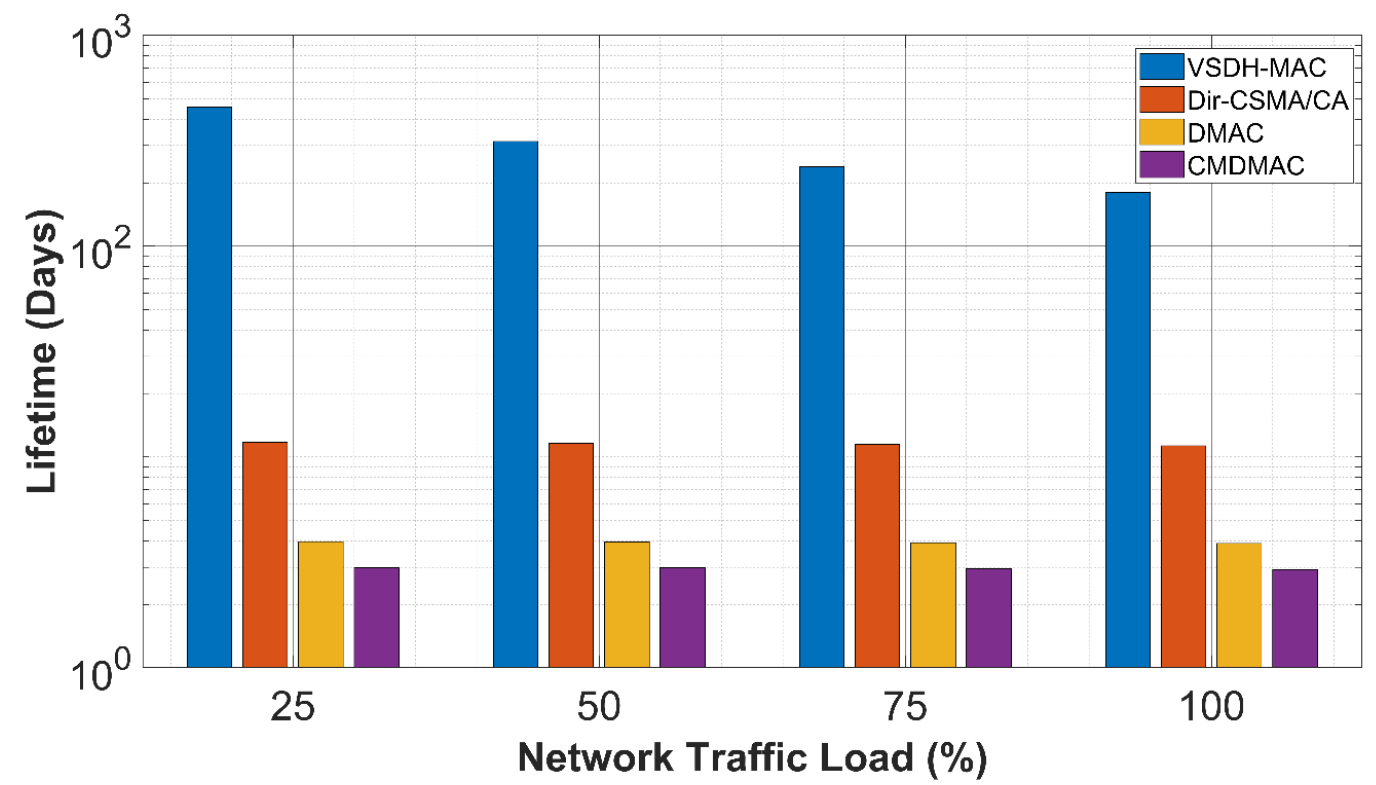

Figure 9. Comparison of expected sensor node lifetime with different network traffic load.

Figures 10 and 11 show the fairness of the VSDH-MAC protocol. Figure 10 shows the impact of transmission distance on fairness performance and the effect of the power control strategy. It can be seen that the effect of distance on throughput is much less with the power control strategy, thus increasing the fairness of the network. In wireless communication, increasing the propagation distance would increase the path loss in the transmission which may cause the SINR to decrease with distance. However, the power control strategy in VSDH-MAC provides a uniform SINR for all sensor nodes regardless of the propagation distance, thus increasing the per node fairness.

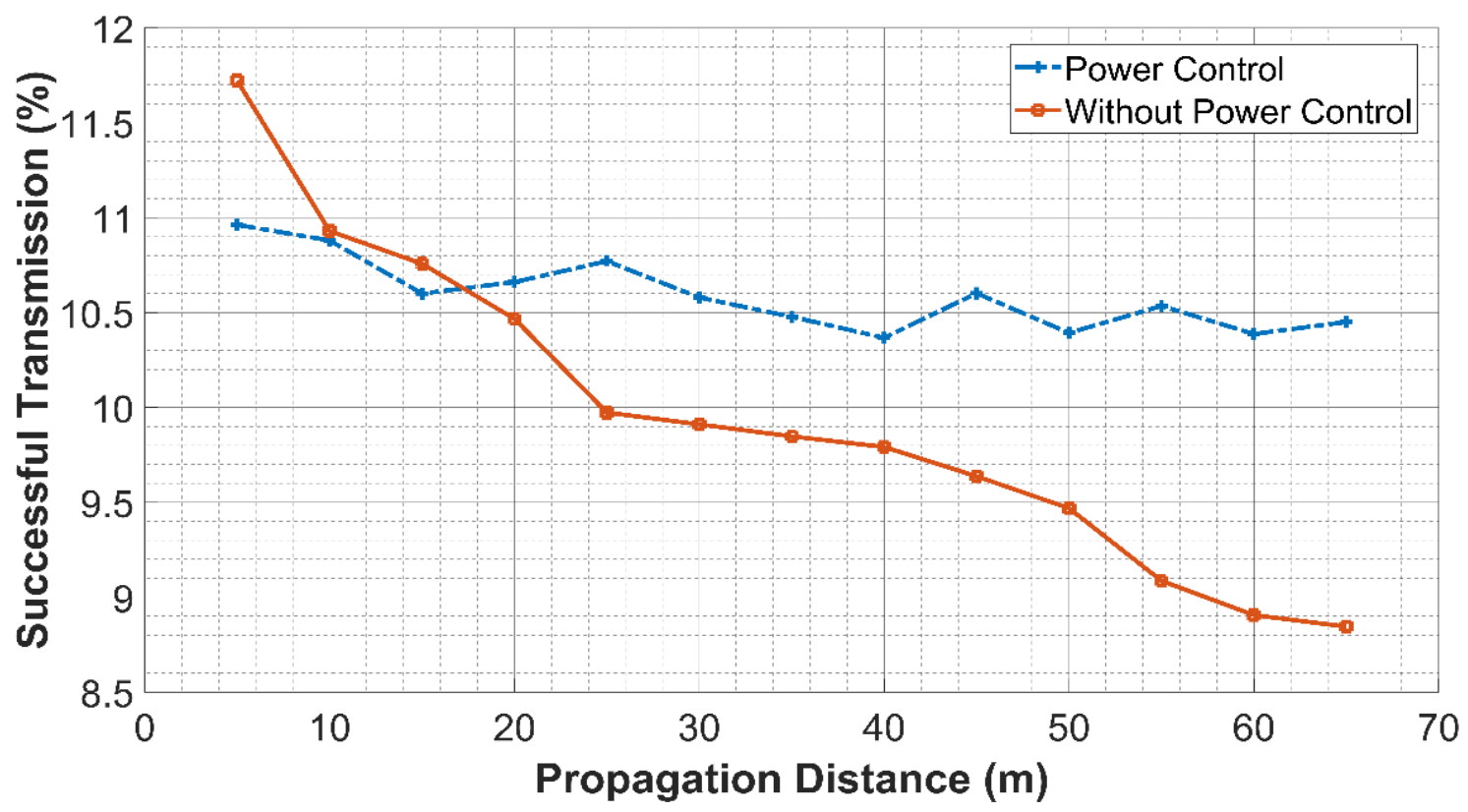

Figure 10. The proportion successful transmissions as a function of distance from the hub at maximum throughput. 


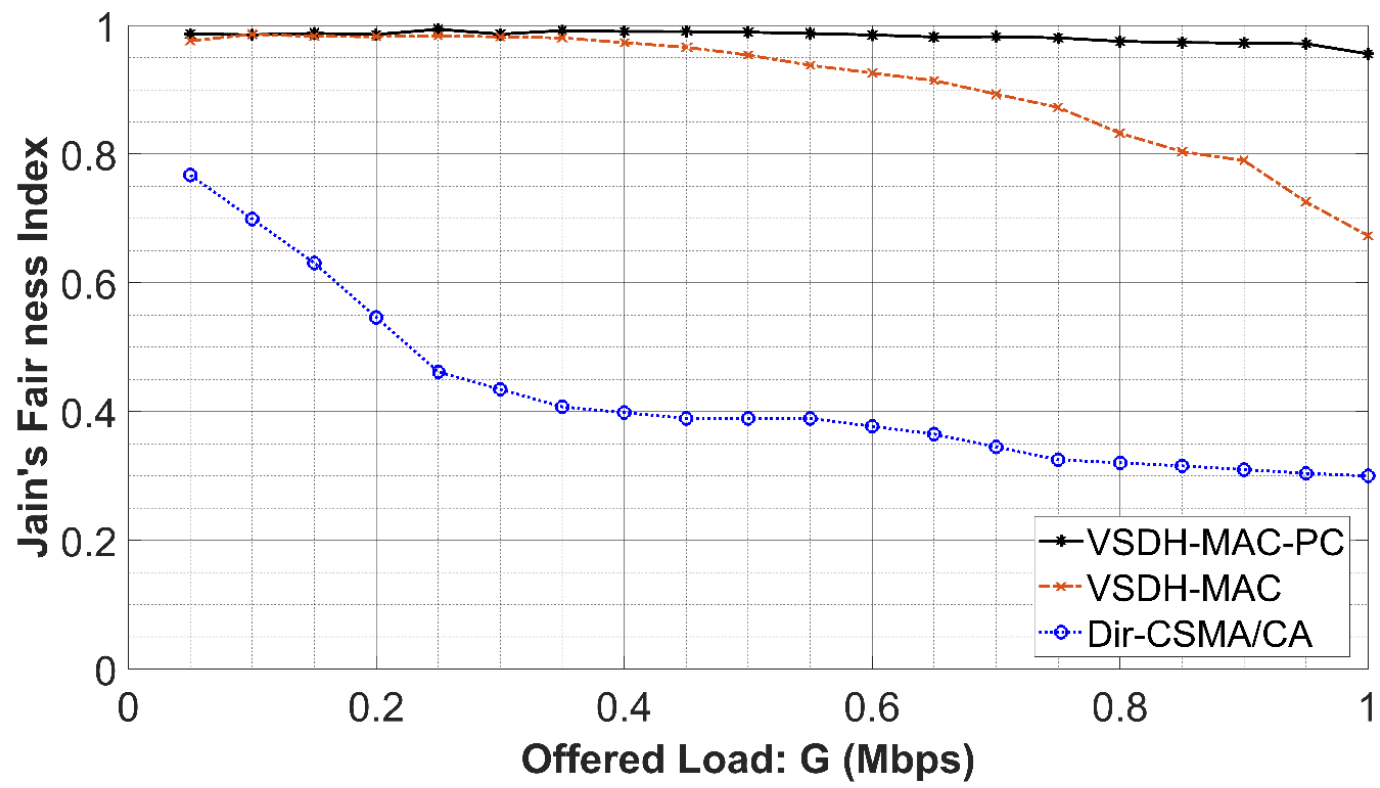

Figure 11. Jain's fairness index improvements with the power control mechanism applied compared to VSDH-MAC with no power control and the modified directional CSMA/CA in a network with 50 nodes.

Figure 11 compares the fairness performance of VSDH-MAC protocol and IEEE 802.11 DCF using Jain's fairness index [27]. Jain's fairness index is used to determine the fairness of the network at different offered loads, and is defined by:

$$
F I=\frac{\left(\sum_{i=0}^{n} x_{i}\right)^{2}}{n \sum_{i=0}^{n} x_{i}{ }^{2}},
$$

where $n$ is the number of nodes in the network, $x_{i}$ is the throughput of the $i$ th node within the network. The fairness index ranges from $\frac{1}{n}$ to 1 . Ideally, when all sensor nodes share the channel equitably, the fairness index should be equal to 1 .

Figure 11 indicates that the VSDH-MAC protocol with the power control strategy achieves a higher fairness index than the case without power control and the directional CSMA/CA. At the low offered load, VSDH-MAC provides a very high Jain's fairness index value. This indicates that all sensor nodes within the network have an equal opportunity to transmit a packet to the hub and of being received successfully. At higher offered load values, the value of the Jain's fairness index decreases, as more nodes try to gain access at a given time and some nodes are forced into back-off.

Since the CSMA/CA protocol is a random access scheme with back-off, it suffers from low fairness performance due to the back-off mechanism. When a sensor node fails to acquire the channel, it will double its back-off window. Under heavy loads, the fairness performance is poor as once a sensor node is able to transmit a packet it will have much better probability of getting access to the channel again than other sensor nodes who might have back-off waiting periods. On the other hand, since the VSDH-MAC performs selective back-off using the CTS SINR threshold, it reduces the number of nodes entering back-off.

Figure 12 shows the relationship between throughput and number of nodes within the network. As the number of nodes in the network increases, the collisions of RTS at the hub increase, sending more nodes into NAV (back-off) hence reducing the throughput. However, as the number of nodes approach a certain threshold, the network throughput levels off to a near constant value. 


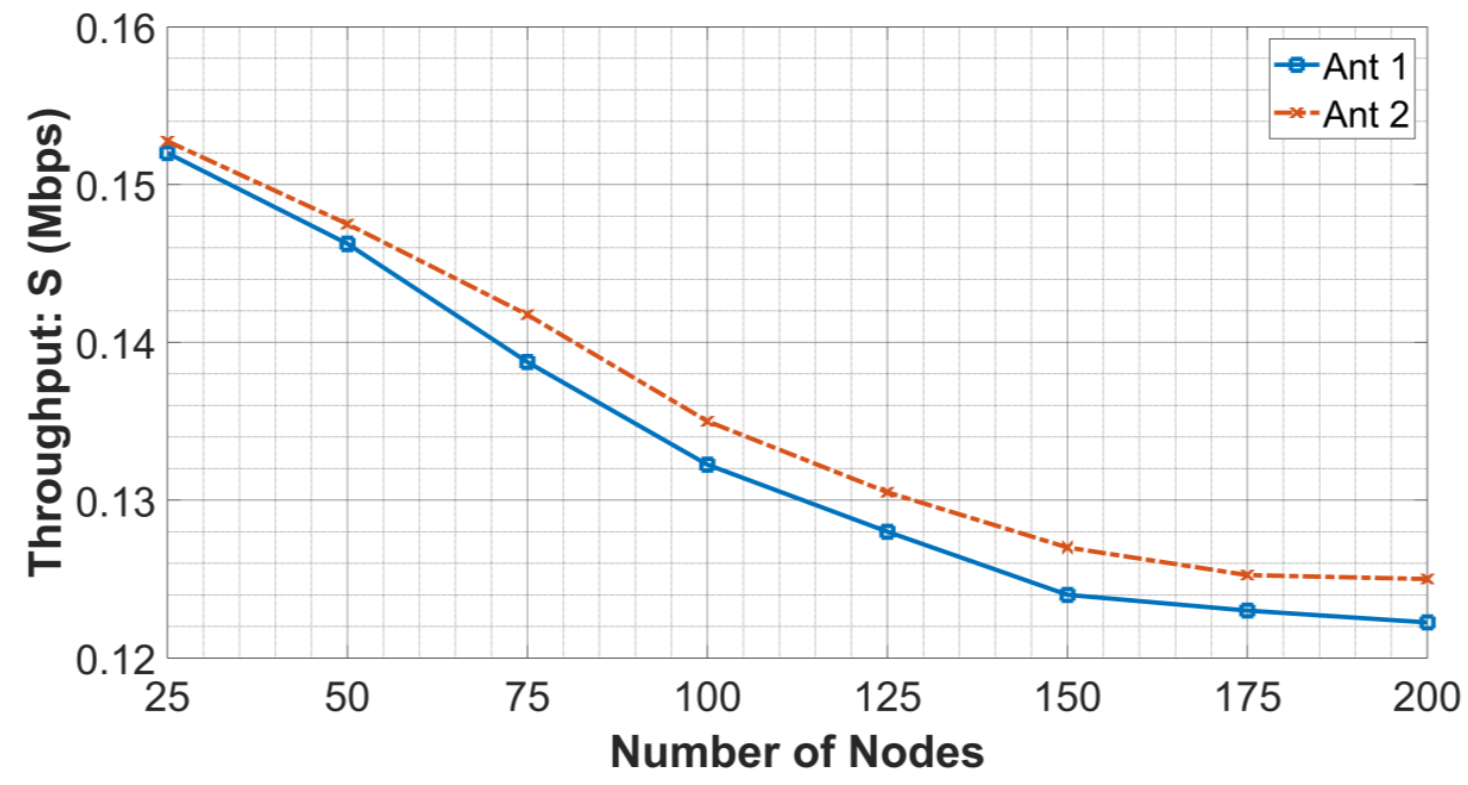

Figure 12. Impact of number of nodes on maximum throughput.

\section{Conclusions}

In this paper, it is shown that the proposed VSDH-MAC and DIFS-VSDH-MAC protocols offer excellent performance in dealing with the trade-off between throughput and node energy consumption. The use of virtual carrier sensing provides the lowest energy consumption, but with a small increase in energy consumption the inclusion of actual carrier sensing provides almost twice the throughput. The major advantage of the VSDH-MAC and DIFS-VSDH-MAC protocols is that they exploit the potential of directional antennas and spatial reuse in achieving high overall network throughput, energy efficiency and improved fairness. It is also worth noting that contention-based protocol tends to have low latency compared to contention-free protocol under low traffic load. Simulation results have shown that the VSDH-MAC protocol is able to provide better throughput and energy efficiency performance than other directional IEEE 802.11 DCF protocols. It should also be noted that we have found the use of real, rather than ideal antenna patterns can make a substantial difference in the network performance, with ideal antennas, the throughput appears to be larger than possible with real antennas, due to the capacity reduction brought about by beam overlap.

Further work is required to consider the performance of the VSDH-MAC and DIFS-VSDH-MAC protocol for mobile WSN scenarios. In addition, three-dimensional (3D) scenarios and the effects of non-line of sight transmission should be considered.

Author Contributions: Conceptualization, A.C., J.D., P.M. and T.H.L.; data curation, A.C.; formal analysis, A.C., J.D. and P.M.; methodology, A.C., J.D., P.M. and T.H.L.; software, A.C. and P.M.; validation, A.C.; writing-original draft, A.C., J.D. and P.M.; writing-review and editing, A.C., J.D., P.M. and T.H.L. All authors have read and agreed to the published version of the manuscript.

Funding: This research received no external funding.

Acknowledgments: The work of T. H. Loh was supported by The 2017-2020 National Measurement System Programme of the UK government's Department for Business, Energy and Industrial Strategy (BEIS), under Science Theme Reference EMT20 of that Programme.

Conflicts of Interest: The authors declare no conflict of interest.

\section{References}

1. Sheng, Z.; Mahapatra, C.; Zhu, C.; Leung, V.C.M. Recent Advances in wireless sensor networks towards efficient management IoT. IEEE Access 2015, 3, 622-637. [CrossRef] 
2. Borges, L.M.; Velez, F.J.; Lebres, A.S. Survey on the Characterization and Classification of Wireless Sensor Network Applications. IEEE Commun. Surv. Tutor. 2014, 16, 1860-1890. [CrossRef]

3. Loh, T.; Liu, K.; Qin, F.; Liu, H. Assessment of the adaptive routing performance of a Wireless Sensor Network using smart antennas. IET Wirel. Sens. Syst. 2014, 4, 196-205. [CrossRef]

4. Zhao, Y.Z.; Mahapatra, C.; Ma, M.; Zhang, J.B.; Leung, C. A survey and projection on medium access control protocols for wireless sensor networks. ACM Comput. Surv. 2012, 45, 7:1-7:37. [CrossRef]

5. Chau, A.; Dawson, J.F.; Mitchell, P.D.; Loh, T.H. Medium access control protocol for wireless sensor networks in Harsh environments with directional antennas. In Proceedings of the 2018 Loughborough Antennas \& Propagation Conference (LAPC 2018), Loughborough, UK, 12-13 November 2018; pp. 1-5.

6. Chau, A.; Dawson, J.F.; Mitchell, P.D. Medium Access and Power Control Protocol for Wireless Sensor Networks with Directional Antennas. In Proceedings of the 10th International Conference on Information and Communication Technology Convergence (ICTC 2019), Jeju Island, Korea, 16-18 October 2019; pp. 582-586.

7. Choudhury, R.R.; Yang, X.; Ramanathan, R.; Vaidya, N.H. Using directional antennas for medium access control in ad hoc networks. In Proceedings of the 8th International Conference on Mobile Computing and Networking, Atlanta, GA, USA, 23-28 September 2002; pp. 59-70.

8. Zhuochuan, H.; Chien-Chung, S.; Srisathapornphat, C.; Jaikaeo, C. A busy-tone based directional MAC protocol for ad hoc networks. In Proceedings of the MILCOM 2002, Anaheim, CA, USA, 7-10 October 2002; Volume 1232, pp. 1233-1238.

9. Nasipuri, A.; Ye, S.; You, J.; Hiromoto, R.E. A MAC protocol for mobile ad hoc networks using directional antennas. In Proceedings of the IEEE Wireless Communications and Networking Conference, Chicago, IL, USA, 23-28 September 2000; pp. 1214-1219.

10. Abdullah, A.A.; Cai, L.; Gebali, F. DSDMAC: Dual Sensing Directional MAC Protocol for Ad Hoc Networks with Directional Antennas. IEEE Trans. Veh. Technol. 2012, 61, 1266-1275. [CrossRef]

11. Choudhury, R.R.; Yang, X.; Ramanathan, R.; Vaidya, N.H. On designing MAC protocols for wireless networks using directional antennas. IEEE Trans. Mob. Comput. 2006, 5, 477-491. [CrossRef]

12. Gang, W.; Peng, X.; Wenming, L. A novel MAC protocol for wireless network using multi-beam directional antennas. In Proceedings of the 2017 International Conference on Computing, Networking and Communications (ICNC), Santa Clara, CA, USA, 26-29 January 2017; pp. 36-40.

13. Duc Ngoc Minh, D.; Huong Tra, L.; Hyo Sung, K.; Choong Seon, H.; Jongwon, C. Multi-channel MAC protocol with Directional Antennas in wireless ad hoc networks. In Proceedings of the 2015 International Conference on Information Networking (ICOIN), Siem Reap, Cambodia, 12-14 January 2015; pp. 81-86.

14. Huang, F.; Leung, K.; Li, V.O.K. Transmission Radius Control in Wireless Ad Hoc Networks with Smart Antennas. IEEE Trans. Commun. 2010, 58, 2356-2370. [CrossRef]

15. Mahmud, M.T.; Rahman, M.O.; Hassan, M.M. Two-Dimensional Cooperation-based Asynchronous Multichannel Directional MAC Protocol for Wireless Networks. In Proceedings of the IEEE Region 10 Conference (TENCON), Jeju Island, Korea, 28-31 October 2018; pp. 1033-1038.

16. Xu, H.; Lei, L. A MAC protocol based on busy tone for ad-hoc networks with beamforming antennas. In Proceedings of the 3rd International Conference on Systems and Informatics (ICSAI), Shanghai, China, 19-21 November 2016; pp. 659-663.

17. Abo-Zahhad, M.; Farrag, M.; Ali, A.; Amin, O. An energy consumption model for wireless sensor networks. In Proceedings of the 5th International Conference on Energy Aware Computing Systems \& Applications, Cairo, Egypt, 24-26 March 2015; pp. 1-4.

18. Agarwal, V.; DeCarlo, R.A.; Tsoukalas, L.H. Modeling Energy Consumption and Lifetime of a Wireless Sensor Node Operating on a Contention-Based MAC Protocol. IEEE Sens. J. 2017, 17, 5153-5168. [CrossRef]

19. Le, T.N.; Pegatoquet, A.; Huy, T.L.; Lizzi, L.; Ferrero, F. Improving Energy Efficiency of Mobile WSN Using Reconfigurable Directional Antennas. IEEE Commun. Lett. 2016, 20, 1243-1246. [CrossRef]

20. Sakakibara, K.; Nakagawa, H.; Yamakita, J. Analysis of energy consumption of IEEE 802.11 DCF under non-saturation conditions. In Proceedings of the 2006 1st International Symposium on Wireless Pervasive Computing, Phuketm, Thailand, 16-18 January 2006; p. 6.

21. Bianchi, G. Performance analysis of the IEEE 802.11 distributed coordination function. IEEE J. Sel. Areas Commun. 2000, 18, 535-547. [CrossRef]

22. RiverBed. Available online: https://www.riverbed.com/ (accessed on 27 July 2020). 
23. Haitao, L.; Gao, S.; Tian Hong, L.; Fei, Q. Low-cost intelligent antenna with low profile and broad bandwidth. IET Microw. Antennas Propag. 2013, 7, 356-364.

24. Takai, M.; Martin, J.; Bagrodia, R.; Ren, A. Directional virtual carrier sensing for directional antennas in mobile ad hoc networks. In Proceedings of the 3rd ACM International Symposium on Mobile ad hoc Networking \& Computing, Lausanne, Switzerland, 9-11 June 2002; pp. 183-193.

25. Wang, Y.; Motani, M.; Garg, H.K.; Chen, Q.; Luo, T. Cooperative Multichannel Directional Medium Access Control for Ad Hoc Networks. IEEE Syst. J. 2017, 11, 2675-2686. [CrossRef]

26. Crossbow Technology Inc. MICAz Datasheet Document, Part Number: 6020-0060-04 Rev A. Available online: http://www.openautomation.net/uploadsproductos/micaz_datasheet.pdf (accessed on 27 July 2020).

27. Jain, R.K.; Hawe, W. A Quantitative Measure Of Fairness And Discrimination For Resource Allocation In Shared Computer Systems. In Technical Report TR-301, Digital Equipment Corporation (DEC) Research; DEC Research: Maynard, MA, USA, 1984.

(C) 2020 by the authors. Licensee MDPI, Basel, Switzerland. This article is an open access article distributed under the terms and conditions of the Creative Commons Attribution (CC BY) license (http://creativecommons.org/licenses/by/4.0/). 